\title{
La variabilité des performances animales en élevage porcin : description et implications pratiques
}

\author{
N. QUINIOU ${ }^{1}$, L. BROSSARD ${ }^{2,3}$, J. VAN MILGEN ${ }^{2,3}$, Y. SALAÜN ${ }^{1}$, H. QUESNEL $L^{2,3}$, \\ F. GONDRET 2,3, J.-Y. DOURMAD 2,3 \\ ${ }^{1}$ IFIP Institut du Porc, Pôle Techniques d'Elevage, BP 35104, F-35651 Le Rheu cedex, France \\ 2 INRA, UMR1348 Physiologie, Environnement et Génétique pour l'Animal et les Systèmes d'Elevage, \\ F-35590 Saint-Gilles, France \\ ${ }^{3}$ Agrocampus Ouest, UMR Physiologie, Environnement et Génétique pour l'Animal et les Systèmes d'Elevage, \\ F-35590 Saint Gilles, France \\ Courriel : nathalie.quiniou@ifip.asso.fr
}

Subie, combattue ou maîtrisée ? La variabilité individuelle se répercute sur la prolificité générale du troupeau quand elle concerne les truies et sur le rendement économique de l'engraissement quand elle concerne les porcs en croissance ${ }^{1}$. Ces dernières années, l'augmentation importante de la prolificité des truies a accentué la variabilité des caractéristiques corporelles des animaux dès le début du développement utérin puis au long de leur vie.

$\mathrm{Au}$ sein d'un élevage porcin, les truies reproductrices, d'une part, et les porcelets produits, d'autre part, sont le plus souvent issus d'un même croisement génétique. Un élevage naisseurengraisseur rassemble à un moment donné des cohortes d'animaux à des stades physiologiques différents pour les truies et à des stades de croissance différents pour les porcelets et les porcs à l'engrais. La conduite en bandes consiste à regrouper dans une même salle les animaux qui sont à un stade similaire afin de réduire la diversité des états au sein de chaque groupe. Ceci présente de nombreux avantages en termes d'organisation du travail de l'éleveur et de gestion de la santé des animaux. Pour autant, même quand ils sont issus d'un type génétique donné et élevés dans des conditions de logement et de statut sanitaire similaires, tous les animaux n'ont pas le même niveau de performances. Une variabilité est ainsi observée à tous les stades de la vie de l'animal. Elle est le plus souvent subie par l'éleveur, mais elle peut aussi être maîtrisée par la mise en œuvre de conduites d'élevage, d'alimentation ou d'abattage, adaptées aux caractéristiques des différents individus.

Selon l'importance des écarts observés au sein d'une même population, chaque caractère ou performance est qualifié de plus ou moins variable. L'adjectif variable est défini en langue française par: «qui prend plusieurs valeurs selon les cas individuels, ou selon les circonstances» (Petit Robert, 1990); à ne pas confondre avec hétérogène «qui est composé d'éléments de nature différente». La variabilité peut alors être quantifiée par une «grandeur qui mesure l'ampleur des variations d'un caractère». L'objectif de cet article est d'examiner les différentes étapes de l'élevage du porc auxquelles s'exprime la variabilité et de décrire ses conséquences, et certaines des pratiques adoptées en élevage afin de mieux la maîtriser.

\section{1 / Variabilité de la taille de la portée}

En 2010, la sélection génétique pour une meilleure prolificité et les évolutions de la conduite d'élevage permettent de sevrer en moyenne 28,2 porcelets par truie productive et par an (Gestion Technique des Troupeaux de Truies (GTTT) ; IFIP, 2011), contre 16,4 il y a 40 ans. Toutefois, le bénéfice de l'hyperprolificité à la naissance ne se retrouve pas en intégralité au sevrage (figure 1) en raison de l'augmentation des pertes de porcelets en maternité.

Figure 1. Evolution de la taille de la portée au cours des quarante dernières années (résultats nationaux GTTT, IFIP).

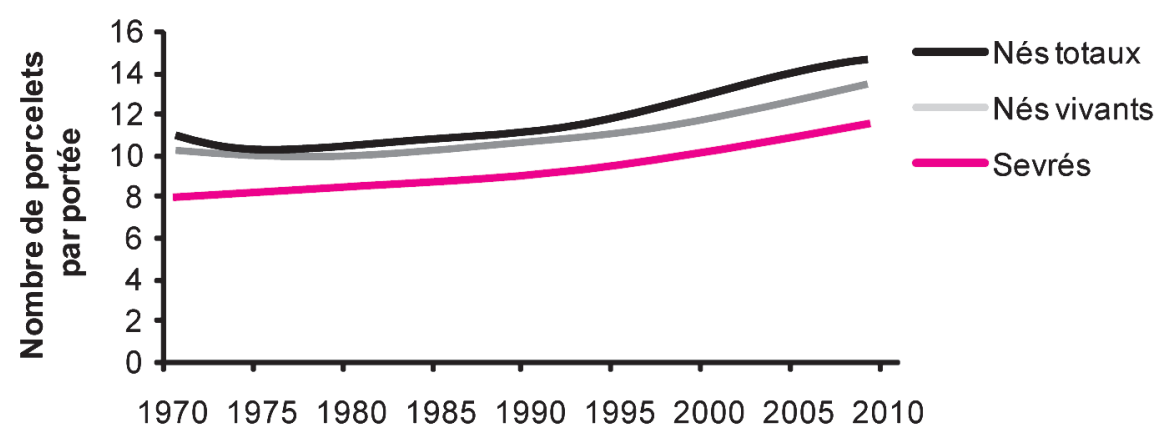

Année

\footnotetext{
${ }^{1}$ Cette présentation fait suite à une réunion de la Commission Porcine de l'INRA consacrée au thème de «la variabilité en élevage porcin». Les auteurs sont membres de l'Unité Mixte Technologique Ingénierie des systèmes de production porcine.
} 
Figure 2. Evolution de la taille moyenne de la portée et de sa variabilité (Ecart-Type) par bandes de 24 truies (données recueillies sur 130 bandes à la station IFIP de Romillé entre 1998 et 2007).

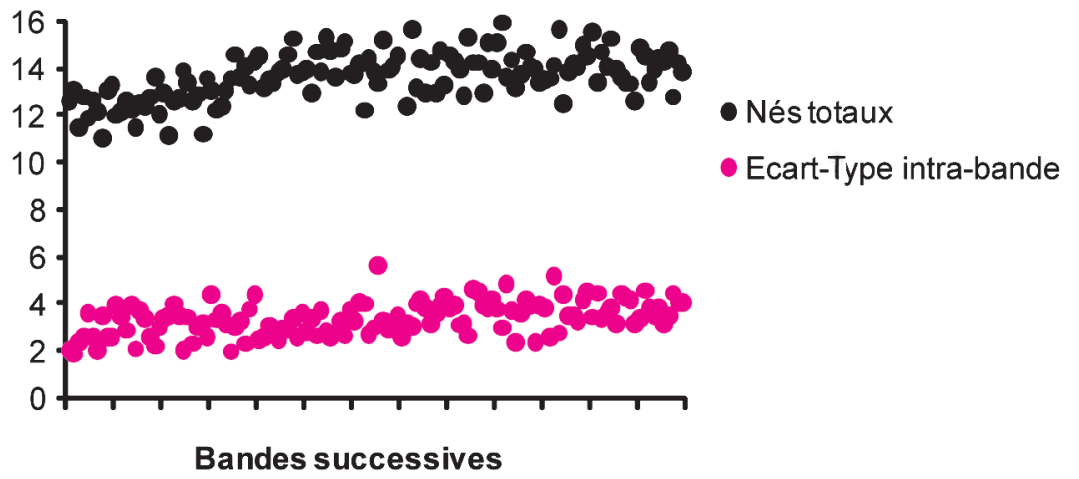

\section{1 / Variabilité entre bandes du troupeau}

Dans un troupeau donné, la taille de portée à la naissance diffère d'une bande de truies à l'autre et entre truies d'une même bande. La figure 2 permet de visualiser les variations de la taille de portée moyenne par bande au sein d'un troupeau. Les conséquences de cette variabilité dépendent du niveau moyen de prolificité du troupeau. En effet, dans un troupeau moyennement prolifique, ce sont les bandes qui présentent une taille de portée basse qui posent problème. Dans ce cas, le nombre de porcelets nés est insuffisant au regard des capacités d'engraissement de l'élevage, le problème étant accru par le fait que tous les porcelets nés vivants ne survivent pas jusqu'au sevrage. Au contraire, dans un troupeau présentant en moyenne une forte prolificité, ce sont les bandes ayant une taille de portée élevée qui posent problème. Dans ce cas, le nombre total de porcelets nés vivants peut dépasser les capacités d'allaitement de l'ensemble des truies de la bande. En effet, les truies ont le plus souvent 14 tétines et qui ne sont pas toujours toutes fonctionnelles. La mise en œuvre de pratiques d'adoption de porcelets (cf. $\S 1.2$ ) entre truies n'est donc plus suffisante; les porcelets présents au-delà du nombre de tétines fonctionnelles sont alors désignés comme «surnuméraires» et requièrent des soins et des installations spécifiques (permettant par exemple un sevrage précoce).

\section{2 / Gestion de la variabilité entre truies d'une même bande}

Les écarts de prolificité entre truies d'une même bande sont gérés par des adoptions. L'éleveur intervient ainsi pour adapter la taille de portée à la capacité d'allaitement de la truie (simplement estimée par le nombre de tétines fonctionnelles) en faisant adopter les porcelets surnuméraires par les truies les moins prolifiques de la bande. Cette pra- tique suppose de prendre en compte à la fois la date de mise bas de la mère et celle de la truie nourrice : en effet, il est conseillé aux éleveurs (Manuel d'application GBPH 2009) de maintenir le porcelet au minimum $6 \mathrm{~h}$ sous sa mère avant de le faire adopter par une truie qui a mis bas depuis moins de $48 \mathrm{~h}$. L'objectif est, d'une part, que le porcelet puisse consommer du colostrum de qualité immunologique élevée (celui de sa mère) dans les heures qui suivent sa naissance et, d'autre part, qu'il puisse également en obtenir de la truie nourrice afin d'acquérir un statut sanitaire compatible avec celui des autres porcelets nés de cette truie (I. Corrégé, communication personnelle). Ces précautions sont importantes car le porcelet naît dépourvu de protection immunitaire et dépend de l'ingestion du colostrum pour acquérir une immunité passive. Toutefois, il n'est pas toujours possible en pratique de respecter ces objectifs, en particulier pour les dernières mises bas de la bande. Afin de grouper les naissances sur une période de temps plus restreinte, de nombreux éleveurs (plus de $80 \%$ d'après une enquête réalisée en 2004 ; Boulot et al 2005) synchronisent le déclenchement des mises bas par l'injection d'une prostaglandine F2 $\alpha$ de synthèse (la PGF $2 \alpha$ endogène initie naturellement le processus de parturition). La mise bas débute alors environ $24 \mathrm{~h}$ après l'injection. Toutefois, même après induction, il arrive que plusieurs jours séparent les mises bas d'une même bande de truies, notamment en relation avec la variabilité de la durée de gestation (de moins de 112 à 116 jours, pour une moyenne à 114 jours ; Sasaki et Koketsu 2007) et l'étalement dans le temps des dates de fécondation. Les porcelets nouveau-nés sont alors susceptibles d'être adoptés par des truies ayant mis bas depuis 3 ou 4 jours. Outre les conséquences sur la protection immunitaire du porcelet, le comportement d'allaitement de la truie peut alors être perturbé par l'arrivée tardive de ces porcelets (Orgeur et al 2002).

\section{2 / Variabilité du poids de naissance intra-portée}

\section{1 / Quelques facteurs d'in- fluence}

Le porcelet naît avec un poids moyen de $1,4 \mathrm{~kg}$, mais cette valeur varie fortement selon les génotypes, les portées, les individus au sein d'une même portée, etc. En 2008, les bases de données issues des troupeaux expérimentaux de l'INRA à St-Gilles et de l'IFIP à Romillé en Bretagne ont été utilisées afin de rechercher les caractéristiques des truies et des portées susceptibles d'influencer la variabilité du poids de naissance (Quesnel et al 2008). Dans ces deux stations, les nouveau-nés sont pesés dans les $24 \mathrm{~h}$ qui suivent la naissance et les mort-nés sont pesés collectivement (INRA) ou individuellement (IFIP).

L'amélioration de la prolificité s'est accompagnée d'une augmentation de la variabilité du poids de naissance, tant chez les truies Large White que chez les croisées Landrace $\times$ Large White (Quesnel et al 2008). Afin de prendre en compte les écarts d'échelle éventuels, la variabilité du poids de naissance est exprimée par son Coefficient de Variation (CV), c'est à dire l'Ecart-Type (ET) exprimé en pourcentage du poids moyen. Le CV est en moyenne de $21 \%$. Il est significativement influencé par la taille et le rang de portée : quand le nombre de nés totaux par portée augmente de moins de 10 porcelets à plus de 15 , le coefficient de variation augmente de 15 à 24\% (tableau 1). De façon concomitante, la proportion de petits porcelets, c'est-à-dire ceux pesant $25 \%$ de moins que le poids moyen dans la portée, augmente.

En moyenne, le coefficient de variation du poids de naissance est moins élevé chez les truies les plus jeunes (autour de $20 \%$ en première et deuxième portées) et augmente ensuite progressivement avec le rang de portée. Toutefois, un CV élevé du poids de naissance à la mise bas «n» n'implique pas un $\mathrm{CV}$ élevé à la mise bas «n $+1 »$. En effet, la répétabilité du CV du poids de naissance a été estimée par Quesnel et al (2008) à 0,10 chez les truies Large White du troupeau de Saint-Gilles, et à 0,12 ou 0,15 , respectivement pour les truies croisées Large White $\times$ Landrace des troupeaux de Romillé et de SaintGilles. Ces valeurs sont proches de celles rapportées chez des truies Landrace par Wittenburg et al (2011).

La taille de la portée et le rang de portée de la truie, associés à l'année de naissance de la truie et à la saison de 
Tableau 1. Evolution des caractéristiques du poids de naissance selon la taille de la portée (Quesnel et al 2008).

\begin{tabular}{|l|c|c|c|c|c|c|}
\hline Classe de taille de la portée (nés totaux) & $\leq 9$ & $10-11$ & $12-13$ & $14-15$ & $\geq 16$ & Effet Taille $^{(1)}$ \\
\hline Poids moyen $(\mathrm{kg})$ & 1,88 & 1,67 & 1,57 & 1,48 & 1,38 & $* * *$ \\
\hline Coefficient de variation (\%) & 15 & 18 & 21 & 22 & 24 & $* * *$ \\
\hline $\begin{array}{l}\text { Pourcentage de porcelets de la portée dont le } \\
\text { poids est inférieur à } 0,75 \text { fois le poids moyen }(\%)\end{array}$ & 6 & 9 & 12 & 13 & 16 & $* * *$ \\
\hline
\end{tabular}

$(1)^{* * *}: P<0,001 ;{ }^{* *}: P<0,01 ;{ }^{*}: P<0,05 ; n s: P>0,10$.

conception, n'expliquent cependant que $20 \%$ de la variabilité du poids de naissance des porcelets. Des recherches complémentaires à partir des données de l'IFIP mettent en évidence une corrélation positive entre la variabilité du poids de naissance et la quantité de lard dorsal déposée par la truie pendant la gestation. Toutefois, dans ce troupeau, les truies qui déposent le plus de gras pendant la gestation sont celles qui en ont mobilisé le plus pendant la lactation précédente. La relation entre variabilité du poids de naissance et gain d'épaisseur de lard pendant la gestation pourrait donc refléter les conséquences du statut métabolique de la truie pendant la lactation sur la qualité des ovocytes et des follicules qu'elle produit lors du cycle suivant (Quesnel et al 2008).

\section{2 / Conséquences sur la survie des porcelets}

\section{a) Survie périnatale}

D'après Zaleski et Hacker (1993), le poids de naissance n'est pas un facteur de risque de mortinatalité. Cette mortalité en cours de mise bas est majoritairement affectée par un rang de naissance élevé, des intervalles longs entre naissances, la rupture précoce du cordon, etc. (Herpin et al 1996), facteurs qui peuvent néanmoins compromettre d'autant plus la survie des porcelets les plus petits. Parmi les porcelets déclarés mort-nés, certains ont respiré avant de mourir (faux mort-nés) et seul le test de flottaison du poumon permet de les discriminer des vrais mort-nés qui meurent pendant l'expulsion. Quand ce test n'est pas fait, la confusion entre vrais et faux mort-nés conduit à constater un taux de mortinatalité particulièrement élevé chez les porcelets de faible poids à la naissance (Leenhouwers et al 1999, Quiniou et al 2002) : $24 \%$ pour les porcelets de moins de $600 \mathrm{~g}, 11 \%$ pour des porcelets pesant entre 600 et $800 \mathrm{~g}$ contre $7 \%$ pour les porcelets qui pèsent plus de $1 \mathrm{~kg}$. Wittenburg et al (2011) ont ainsi récemment recommandé de considérer le nombre total de porcelets nés par portée (et de ne pas omettre les mort-nés) pour analyser de façon plus précise les variations de poids à la naissance.

Dans les 24 à 48 h qui suivent la naissance, les pertes de porcelets sont principalement observées parmi les nouveau-nés qui pèsent moins de $1 \mathrm{~kg}$. Les causes de mortalité répertoriées pour les porcelets les plus chétifs sont principalement des écrasements ou un état de faiblesse générale, ce dernier étant l'une des raisons principales entraînant les écrasements (Le Cozler et al 2000). En outre, le faible poids de naissance a été identifié comme le facteur de risque le plus important d'un mauvais rétablissement de la température corporelle consécutivement à l'hypothermie en période périnatale (Kammersgaard et al 2011).

\section{b) Survie post-natale}

Les trois quarts de la mortalité postnatale interviennent dans les 7 jours qui suivent la naissance et le risque de mortalité augmente quand le poids de naissance diminue (Edwards et Malkin 1986, Marchant et al 2000). Ainsi, une fois les adoptions terminées (vers $24 \mathrm{~h}$ d'âge), un porcelet qui pèse moins de $600 \mathrm{~g}$ à la naissance a environ 150 fois plus de risque de mourir avant 7 jours qu'un porcelet qui pèse plus de $1,6 \mathrm{~kg}$ à la naissance ; ce facteur de risque relatif de mourir étant réduit à 34 pour 1 et à 10 pour 1 , pour ceux qui pèsent respec- tivement entre 0,6 et $0,8 \mathrm{~kg}$ ou entre 0,8 et $1,0 \mathrm{~kg}$ (figure 3 ). Le risque de mourir avant le sevrage est d'autant plus important pour les porcelets les plus petits que le CV du poids de naissance dans la portée est important (Milligan et al 2002).

A partir de l'étude des liaisons génétiques entre critères, Huby et al (2003) montrent que la survie des porcelets en maternité est génétiquement liée à la variabilité du poids dans les portées. D'après ces travaux et ceux de Knol et al (2002) et de Damgaard et al (2003), une sélection dite canalisante sur ces critères, c'est-à-dire réalisée non seulement sur leur moyenne mais également sur leur variabilité, devrait permettre d'améliorer la survie des porcelets avant le sevrage. Une telle sélection dans l'espèce lapin s'avère par exemple satisfaisante pour réduire la variabilité des poids de naissance et améliorer la survie des jeunes (Garreau et al 2008). Une autre piste pour réduire la variabilité des poids de portées serait de moduler l'alimentation de la truie. Toutefois, les stratégies testées jusqu'à présent pendant la gestation ont permis d'améliorer la vitalité des porcelets (Quiniou 2005, Quiniou et al 2008), mais n'ont pas eu d'effet sur la variabilité des poids de naissance (Campos et al 2011). En revanche, van den Brand et al (2006) ont montré que l'alimentation de la truie avant la fécondation pouvait influencer la variabilité intra-portée des

Figure 3. Facteur de risque de mortalité après les adoptions selon le poids à la naissance (Quiniou et al 2002).

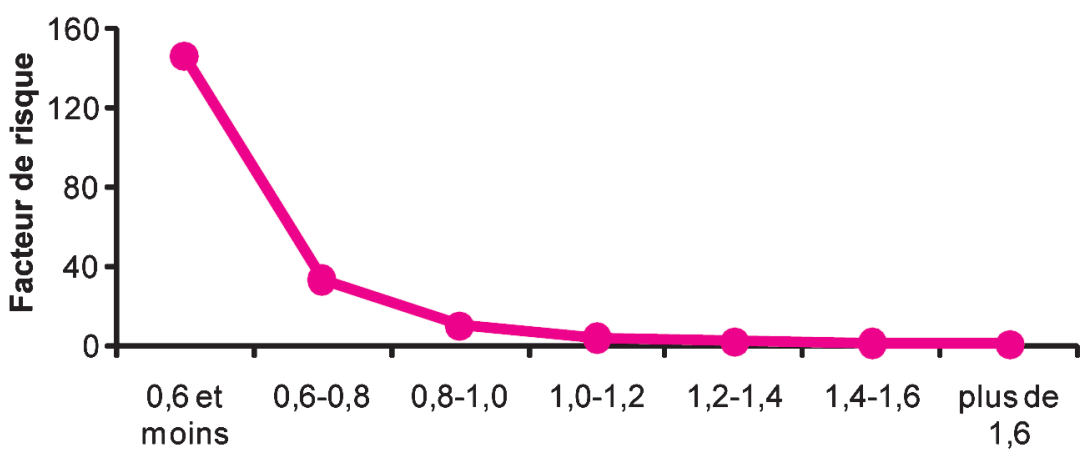

Classe de poids de naissance $(\mathrm{kg})$ 
poids de naissance (réduction du CV de 4 points), mais sans répercussion sur la survie précoce des porcelets.

\section{c) Survie après le sevrage}

En post-sevrage et en engraissement aussi, les pertes sont généralement plus importantes chez les porcelets les plus légers à la naissance. Les pertes après le sevrage chez ces porcelets sont observées pour un tiers d'entre elles en postsevrage (période de 6 semaines environ préparant l'engraissement) et pour deux tiers d'entre elles en phase d'engraissement (Le Cozler et al 2004). Les pertes après le sevrage ont notamment lieu lors des changements de bâtiments. Or, l'entrée en post-sevrage ou en engraissement est réalisée le même jour pour tous les porcs d'un âge donné, afin de respecter les contraintes de la conduite en bandes. D'après Le Cozler et al (2004), le statut sanitaire, en particulier l'apparition de pathologies digestives, explique la plupart des pertes après le sevrage, mais ceci quel que soit le poids de naissance. Dans une étude conduite aux Etats-Unis, il a d'ailleurs été montré que le poids de naissance exerçait un effet linéaire sur la survie pré- et post-sevrage comme dans le cadre français, mais n'affectait pas la probabilité de survie durant la croissance-finition (Fix et al 2010).

\section{3 / Croissance des porcelets pendant la lactation et après le sevrage}

A chaque stade de croissance, l'écart de poids observé en début de période s'accentue en fin de période. Cependant, cette augmentation n'est pas constante mais dépend du poids initial. Ainsi, en maternité, pour $100 \mathrm{~g}$ de plus à la naissance, le poids de sevrage sera supérieur de $400 \mathrm{~g}$ si le poids de naissance est environ de $1 \mathrm{~kg}$, tandis que le poids de sevrage sera plus élevé de seulement $200 \mathrm{~g}$ si le porcelet pèse environ $1,2 \mathrm{~kg}$ à la naissance (Quiniou et al 2002). Ce résultat amène alors à considérer non plus les écarts en valeur absolue mais en valeur relative.

La vitesse de croissance inférieure observée avant le sevrage chez les porcelets les plus légers à la naissance ne semble pas provenir d'un potentiel de croissance moindre. En effet, plus les porcs sont légers à la naissance, plus leur gain de poids relatif est élevé. Ainsi, le poids des porcelets qui pèsent $1 \mathrm{~kg}$ à la naissance est multiplié par 9 à 28 jours, alors qu'il est multiplié seulement par 5,5 chez ceux qui pèsent plus de 1,8 kg (Quiniou et al 2004). Malgré tout, les premiers restent plus légers au sevrage. Il est probable que la moindre consommation de colostrum et de lait des porcelets chétifs à la naissance ne leur permette pas de réduire l'écart de poids avec les plus lourds. Cependant, Leece (1971, cité par Scheel et al 1977) a mis en évidence un gain de poids comparable entre des porcelets de poids différents à la naissance dès lors que la compétition à la mamelle était supprimée. Les porcelets les plus légers subissent en effet la dominance des plus lourds ; ces derniers accaparent les tétines antérieures, les plus productives (English et Wilkinson 1982).

En post-sevrage, l'influence du poids de naissance sur la vitesse de croissance a été clairement démontrée (Quiniou et al 2002, Beaulieu et al 2010). En revanche, à notre connaissance aucune donnée n'est disponible dans la bibliographie permettant de quantifier l'effet direct du poids de naissance sur la quantité d'aliment ingérée selon le poids de naissance. En effet, les porcs sont le plus souvent élevés en groupe durant cette période. De façon indirecte, Bruininx et al (2001) montrent cependant que le niveau d'ingestion individuelle moyenne est plus faible pour les porcelets légers au sevrage que pour les porcelets plus lourds, chez des animaux logés en groupe. Mais les écarts de poids au sevrage ne s'expliquent que partiellement par le poids de naissance. En outre, Lynch et al (2006), observent chez des porcelets élevés en loges individuelles que les animaux légers à la naissance consomment moins d'aliment que les animaux plus lourds sur l'ensemble de la période de post-sevrage. Dans ces deux essais, il est intéressant de noter que les auteurs rapportent une ingestion plus élevée chez les porcelets les plus légers seulement de façon transitoire, pendant que les porcelets les plus lourds sont occupés à établir la hiérarchie dans la case (Brouns et Edwards 1994).

En engraissement, Quiniou et al (2004) ont étudié des groupes de porcs issus de la même portée et choisis, à l'entrée en engraissement, selon leur poids à la naissance : parmi les plus légers, les plus lourds ou ceux de poids moyen. Ces auteurs montrent que les porcs les plus légers à la naissance présentent des niveaux d'ingestion plus faibles. Toutefois l'efficacité d'utilisation de l'aliment mesurée sur une même durée d'engraissement est comparable à celle observée chez les porcs plus lourds. Ce résultat confirme donc le rôle du facteur «aliment ingéré» comme frein de la croissance, tout comme en maternité pour la quantité de lait. Quand les porcs sont élevés en loges individuelles et ne subissent de fait aucune compétition à l'auge, Gondret et al (2006) montrent qu'ils ingèrent la même quantité d'aliment quel que soit leur poids de naissance et leur poids d'entrée en engraissement. Dans ces conditions, et pour un même poids d'abattage, ces auteurs rapportent une efficacité alimentaire moindre chez les porcs les plus légers à la naissance.

Les conséquences du poids de naissance sur les caractéristiques de la carcasse dépendent des conditions d'abattage. En effet, lorsque les porcs les plus légers à la naissance sont abattus en même temps que le reste des porcs de la bande afin de libérer la salle d'engraissement dans des délais compatibles avec l'arrivée des porcs de la bande suivante, ils ont des poids d'abattage plus faibles que le reste de la bande (Quiniou et al 2002, Le Cozler et al 2004). Cela correspond à la plupart des situations d'élevage ; les effets du poids d'abattage et du poids de naissance sont donc partiellement confondus.

Les conséquences du faible poids de naissance combiné à la variabilité des portées en période postnatale s'exercent aussi sur la composition des carcasses et la qualité des produits. Les porcelets légers à la naissance ont généralement formé un plus faible nombre de fibres musculaires in utero (Gondret et al 2005, 2006) et présentent une plus faible teneur en gras corporel à la naissance (Morise et al 2009) du fait d'une différenciation adipocytaire retardée in utero (Gondret et al 2011). Ce phénotype pourrait modifier le potentiel de croissance des tissus maigres et gras en postnatal, et la composition finale des tissus. Ainsi, les porcelets les plus légers à la naissance auront en moyenne des fibres musculaires de diamètre plus élevé pour un même poids de muscle au stade d'abattage (Gondret et al 2005, 2006). Dans un cadre expérimental, les porcelets de faible poids ont d'ailleurs pu tirer profit d'une supplémentation précoce en L-carnitine favorisant la myogenèse (Lösel et al 2009) pour atténuer les conséquences sur leur structure musculaire comparativement à leurs congénères de poids de naissance plus élevé. Dans les élevages, le poids de naissance n'influence cependant pas significativement la teneur en viande maigre de la carcasse (tableau 2), que les porcs soient abattus au même poids ou non. En revanche, des différences sur l'adiposité corporelle apparaissent entre les études selon les pratiques de logement et d'alimentation (tableau 3). Dans le cas où les porcs sont alimentés à volonté sans compétition à l'auge (loge individuelle), les carcasses des porcs les plus légers à la naissance sont souvent plus grasses au stade commercial d'abattage (Gondret et al 2006). Cela n'est pas le cas lorsque les porcs sont élevés en groupe en conditions d'alimentation plafonnée (Gondret et al 2005). Il est 
Tableau 2. Comparaison des performances de carcasse selon le poids de naissance pour un poids d'abattage non égalisé.

\begin{tabular}{|c|c|c|c|c|c|c|c|c|}
\hline Alimentation & Poids de naissance $(\mathrm{kg})$ & $<1,0$ & $1,0-1,2$ & $1,2-1,4$ & $1,4-1,6$ & $1,6-1,8$ & $\geq 1,8$ & Effet Poids ${ }^{(4)}$ \\
\hline \multirow[t]{3}{*}{ A volonté } & Age à l'abattage (j) & 164 & 161 & 160 & 157 & 158 & 155 & $* * *$ \\
\hline & Poids d'abattage (kg) & 105 & 108 & 110 & 112 & 114 & 115 & $* *$ \\
\hline & TVM $(\%)$ & 61,0 & 60,8 & 61,2 & 61,2 & 60,4 & 60,4 & ns \\
\hline \multirow[t]{3}{*}{ Rationnée (2) } & Age à l'abattage (j) & 185 & 184 & 182 & 180 & 181 & 177 & $* * *$ \\
\hline & Poids d'abattage (kg) & 101 & 104 & 108 & 110 & 109 & 111 & $* *$ \\
\hline & TVM $(\%)$ & 60,2 & 60,2 & 59,8 & 59,9 & 60,1 & 60,1 & ns \\
\hline
\end{tabular}

(1) Quiniou et al 2004 ; (2) Le Colzer et al 2004 ; (3) Teneur en viande maigre.

(4) ${ }^{* *}: \mathrm{P}<0,001 ;{ }^{* *}: \mathrm{P}<0,01 ;{ }^{*}: \mathrm{P}<0,05$; ns : $\mathrm{P}>0,10$.

alors probable que le potentiel de croissance compensatrice des tissus gras chez les porcs légers à la naissance soit limité par le rationnement relatif induit par leur moindre combativité pour l'accès à l'aliment. Il est aussi possible que l'effet du poids de naissance sur la composition corporelle soit plus marqué chez les femelles (Rehfeldt et al 2008).

\section{4 / Des pratiques d'élevage pour mieux gérer la variabilité}

L'hyperprolificité est maintenant largement répandue dans les élevages, même si elle se traduit comme précédemment évoqué par des portées de poids plus variables à la naissance et, de ce fait, par un nombre et une proportion accrus de petits porcelets. D'après les scénarios envisagés sur la base des per- formances présentées ci-après, les réponses techniques étudiées, en termes de conduite d'élevage, pour la gestion de l'hyperprolificité sont toutes économiquement intéressantes, en comparaison à une situation initiale témoin avec des truies non-hyperprolifiques. Ainsi, même si les porcelets surnuméraires sont plus petits et plus fragiles pendant les premiers jours de vie, il reste toujours intéressant de les sauver pour les engraisser ou les vendre, tant d'un poids de vue économique qu'éthique (Gourmelen et Le Moan 2004).

a) Adapter les soins à la naissance pour compenser le manque de vitalité

L'augmentation de la variabilité des portées à la naissance a amené des éleveurs à consacrer plus de temps aux animaux (Martel et al 2008). Cet accroisse- ment de la présence dans les salles de maternité leur permet de mieux suivre le déroulement de la mise bas et d'apporter des soins spécifiques aux porcelets nouveau-nés qui en ont le plus besoin. Parmi les pratiques courantes, citons le séchage du porcelet pour limiter ses dépenses énergétiques, la mise à la mamelle afin de raccourcir le délai entre la naissance et la première tétée, l'administration de colostrum (prélevé sur les truies de l'élevage) ou de suppléments nutritionnels, etc. Toutefois ces interventions nécessitent du temps de travail et tous les éleveurs ne sont pas disposés à les mettre en œuvre (par exemple la nuit ou lorsque d'autres travaux accaparent l'éleveur sur l'exploitation). Des modifications de la conduite alimentaire de la truie gravide ont donc été étudiées puis validées expérimentalement afin de

Tableau 3. Comparaison des performances de carcasse selon le poids de naissance pour un même poids d'abattage (d'après Gondret et al 2005, 2006).

\begin{tabular}{|c|c|c|c|c|}
\hline Type d'alimentation & Poids de naissance $(\mathrm{kg})$ & $0,75-1,25$ & $1,75-2,05$ & Effet Poids ${ }^{(4)}$ \\
\hline \multirow{4}{*}{$\begin{array}{l}\text { A volonté sans compétition } \\
\text { à l'auge }\end{array}$} & Age à l'abattage (j) & 171 & 159 & $* * *$ \\
\hline & Poids d'abattage $(\mathrm{kg})$ & 112 & 112 & - \\
\hline & TVM $(\%)$ & 61,1 & 63,0 & ** \\
\hline & Epaisseur de lard dorsal (mm) & 18,2 & 15,0 & ** \\
\hline \multirow{4}{*}{$\begin{array}{l}\text { Plafonnée en } \\
\text { engraissement }\end{array}$} & Age à l'abattage (j) & 166 & 154 & $* *$ \\
\hline & Poids d'abattage $(\mathrm{kg})$ & 101 & 103 & - \\
\hline & $\operatorname{TVM}(\%){ }^{(3)}$ & 60,9 & 59,5 & ns \\
\hline & Epaisseur de lard dorsal (mm) & 16,7 & 16.9 & ns \\
\hline
\end{tabular}

(1) Femelles élevées en cases individuelles, issues d'un croisement Piétrain x (Large White x Landrace).

(2) Mâles castrés et femelles issus d'un croisement Large White $x$ Landrace.

(3) Teneur en viande maigre.

(4) ${ }^{* * *}: \mathrm{P}<0,001 ;{ }^{* *}: \mathrm{P}<0,01 ;{ }^{*}: \mathrm{P}<0,05$; ns : $\mathrm{P}>0,10$ 
proposer des solutions permettant d'améliorer la survie des porcelets pendant les premières heures suivant la naissance, de manière à pallier la surveillance moindre. L'un des principaux changements réalisés ces dernières années a consisté à revoir les plans d'alimentation. Les plans d'alimentation constants (une même ration du début à la fin de la gestation) ont été remplacés par des cinétiques qui privilégient des apports importants en début et en fin de gestation pour un même apport global sur 115 jours (Quiniou 2005, IFIP 2008).

b) Prise en compte du poids dans la constitution des cases en engraissement

A l'entrée en post-sevrage ou en engraissement, la bande de porcs est répartie dans des cases dont le nombre est déterminé par la configuration des bâtiments. La constitution des groupes au sein de chaque case intègre de nombreux éléments, tels que l'origine de portée, le type sexuel et le poids vif. La constitution de groupes homogènes par leur poids permet de gérer chaque case de manière spécifique, notamment en termes de plan d'alimentation, tant en post-sevrage qu'en engraissement. Cette mise en lots conditionne également en partie l'évolution de la variabilité du poids au cours de la croissance. Plusieurs études montrent que le coefficient de variation du poids de groupes homogènes (CV initial inférieur à 7\%) en début d'engraissement reste relativement stable ou augmente légèrement au cours de l'engraissement alors que celui de groupes plus hétérogènes (CV initial supérieur à 15\%) diminue (Anil et al 2007, Brossard et al 2011).

Différant de la pratique habituelle de l'élevage en cases de taille assez réduite (8-12 animaux), l'élevage des porcs en croissance en grands groupes (jusqu'à 200 porcs dans la même case) se développe. Cette pratique, qui s'intègre dans une perspective d'élevage de précision, a conduit à l'utilisation de dispositifs de pesée et de tri automatiques qui permettent de suivre l'évolution de la distribution des poids au sein de ces groupes. Ceci permet d'ajuster les plans d'alimentation et d'optimiser la gestion des départs : les animaux présentant le poids recherché peuvent ainsi être séparés automatiquement du groupe.

c) Conduite alimentaire et variabilité de la croissance

La conduite alimentaire impacte la variabilité des performances. Dans une étude de simulation des performances de porcs suivis entre 65 jours d'âge et $110 \mathrm{~kg}$, Brossard et al (2009) ont montré que la variabilité de la vitesse de croissance augmente quand les porcs reçoivent des régimes formulés pour apporter les acides aminés à un niveau inférieur de $10 \%$ au besoin moyen du groupe, cet effet étant plus important quand le nombre de phases alimentaires augmente. A l'inverse, des apports supérieurs au besoin moyen du groupe n'affectent pas la variabilité de la vitesse de croissance.

Il existe aussi des interactions entre la constitution du groupe de porcs et le niveau d'alimentation. Ainsi, Massiot et Quiniou (2008) ont observé que l'homogénéité des porcs est influencée différemment par le sexage (élevage séparé des mâles castrés et des femelles) selon le plan de rationnement. Le poids des porcs rationnés en fin d'engraissement à $2,5 \mathrm{~kg} / \mathrm{j}$ est moins variable avant le premier départ à l'abattoir que celui des porcs rationnés en début d'engraissement puis alimentés à volonté. Le sexage ne réduit pas la variabilité des porcs rationnés en fin d'engraissement. En revanche, il permet de réduire la variabilité des porcs rationnés en début d'engraissement puis alimentés à volonté (pratique visant à sécuriser le démarrage de la croissance en cas de problème sanitaire).

d) Gestion des transitions alimentaires selon le poids

La variabilité du poids à un âge donné est associée à des besoins nutritionnels différents (Brossard et al 2012). L'individualisation des apports alimentaires apparaît alors comme une solution possible pour obtenir des performances moins variables. Des systèmes permettant une alimentation de précision individualisée des porcs élevés en groupe existent déjà (Hauschild et al 2010), mais sont encore à l'état de prototype. En attendant leur mise au point pour une diffusion à plus grande échelle, une alimentation adaptée au poids moyen des individus dans la case peut en revanche être déjà envisagée.

Figure 4. Poids moyen des porcs en fonction de leur poids de naissance le jour d'un changement d'aliment réalisé lorsque le poids moyen de tous les porcs de la bande atteint $65 \mathrm{~kg}$ (résultats issus de trois bandes de porc étudiées à la station IFIP de Romillé).

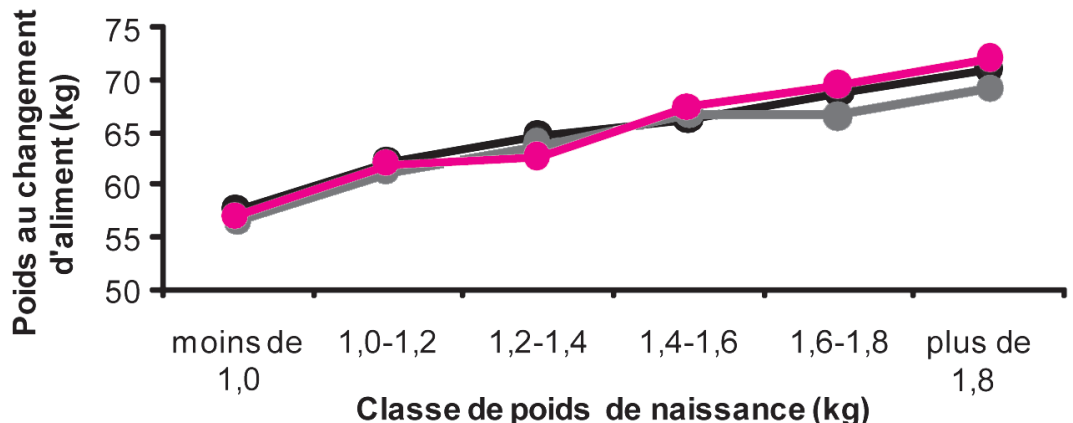

L'une des conduites alimentaires les plus fréquentes en engraissement consiste à distribuer deux aliments, respectivement pendant les périodes de croissance et de finition, différant en particulier par leur teneur en protéines/ acides aminés. Selon le dispositif d'alimentation en place, la transition de l'aliment croissance à l'aliment finition s'effectue soit le même jour soit à des jours différents pour les différentes cases de la bande.

Un changement d'aliment appliqué lorsque tous les porcs de la bande atteignent en moyenne $65 \mathrm{~kg}$ a pour conséquence que les porcs les plus légers à la naissance sont aussi plus légers à ce stade (figure 4) et présentent donc des besoins nutritionnels plus élevés, en particulier en ce qui concerne l'équilibre entre les apports en acides aminés et l'énergie (Noblet et Quiniou 1999). Pour ne pas pénaliser les performances de ces animaux par un apport limitant en acides aminés, il est donc conseillé de regrouper les porcs par gamme de poids à l'entrée en engraissement et de moduler la durée de distribution de l'aliment croissance case par case quand cela est possible. Une alternative est de retarder le passage à l'aliment finition de toute la bande, mais il faut que cette conduite reste compatible avec les exigences de maitrise des rejets d'azote et de phosphore (Corpen 2003) qui impliquent que l'aliment de finition doit représenter au moins $60 \%$ de l'aliment total consommé. En post-sevrage, le même principe prévaut pour la transition entre les aliments de $1^{\text {er }}$ et $2^{\text {ème }}$ âge.

\section{5 / Commercialisation des porcs et valorisation des carcas- ses}

a) Critères de détermination de la valeur économique de la carcasse

Le prix du porc charcutier est déterminé par la somme d'un prix de base, et 
Figure 5. Description graphique de la plus-value appliquée au prix de la carcasse en fonction du poids dans la zone UniporcOuest en 2009 (a) et conséquence sur la plus-value moyenne du lot abattu selon son poids moyen et sa variabilité (ET : EcartType) (b).

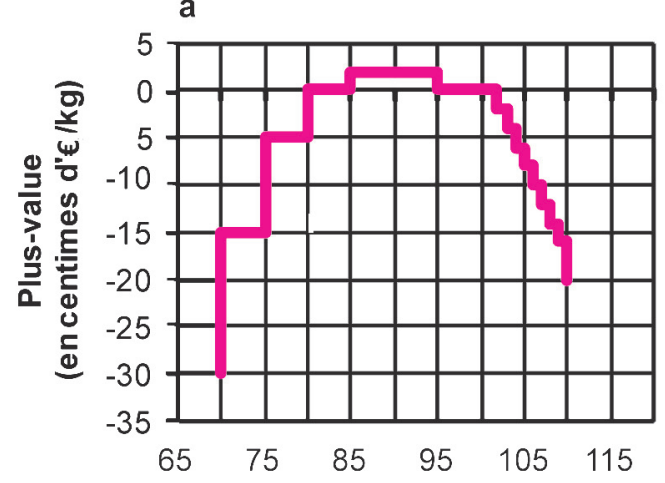

Poids de carcasse $(\mathrm{kg})$

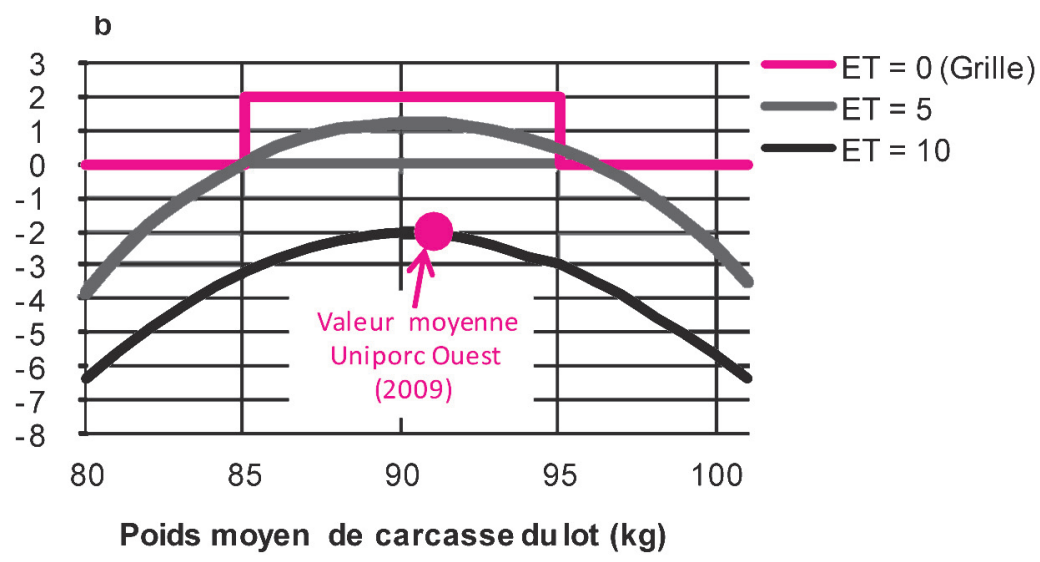

d'un correctif arithmétique intégrant les plus ou moins-values liées au classement de la carcasse selon son poids et sa teneur en viande maigre (www.uniporcouest.com, Marouby et al 2001) remplacée récemment par le Taux de Muscle des Pièces (TMP).

Cette «grille» de paiement rend compte de la demande des utilisateurs (abatteurs et transformateurs) et oriente très puissamment les objectifs des producteurs : en effet, les plus-values tenant compte du TMP progressent fortement jusqu'à la valeur de TMP de $61 \%$, pour plafonner au-delà. De même, les moinsvalues prenant en compte le poids déterminent une plage préférentielle (dite "«cœur de gamme») de poids de carcasse compris entre 85 et $95 \mathrm{~kg}$ (soit, sur la base d'un rendement de carcasse de $76,5 \%$, une plage de poids vif de 111-124 $\mathrm{kg}$, figure 5a) et pénalisent d'autant plus lourdement le prix de la carcasse que son poids s'écarte de cette plage. Cette grille, très discriminante au plan économique, a conduit au fil des années à une augmentation ininterrompue de la teneur en viande maigre, de même qu'à celle du poids de carcasse (le cour de gamme s'étant porté au cours du temps vers des poids plus élevés).

Il est permis de s'interroger sur la pertinence économique de la valeur marginale du point de muscle et du $\mathrm{kg}$, les différents usages de la carcasse en aval (viande fraîche, charcuterie cuite, charcuterie sèche notamment) ayant des exigences spécifiques en termes de poids et de teneur en muscle, alors même que la grille de paiement est unique (du moins pour l'essentiel du marché). Cela signifie que l'adaptation à ces différents usages est confiée quasi-exclusivement au tri opéré par les opérateurs d'aval, ce tri étant lui-même permis par la variabilité des carcasses qui subsiste inter-élevages mais aussi intra-élevages. Il y a donc un paradoxe à décourager toute variabilité à la sortie de l'élevage alors même que seule cette variabilité permet plus en aval de satisfaire des demandes pour des produits très différents. Cette situation rend compte de la position dominante de la transformation en «cuit» (fortement demandeuse de maigre), les autres demandes étant servies de manière «résiduelle», du moins dans le contexte des régions du Grand Ouest de la France.

\section{b) Gestion des carcasses selon leur poids}

Le choix de la date d'abattage des porcs d'une bande résulte principalement d'un compromis entre le nombre de départs possibles et le moment auquel une proportion élevée de porcs atteint le poids d'abattage escompté. De ce fait, en théorie, plus la variabilité des poids en fin d'engraissement est élevée, plus les départs devraient être fractionnés. Cependant, en pratique, l'éleveur est aussi contraint de respecter des tailles de lot minimales à chaque départ, afin d'optimiser la charge des camions de transport, dont la dimension est peu flexible.

Le poids moyen d'abattage recherché tiendra compte du contexte économique défini par la grille de paiement (Rivest 2009). Mais ce poids est aussi une donnée structurelle de l'élevage déterminant (avec la vitesse de croissance et le nombre de bandes) la durée d'occupation d'une salle par une bande (et, audelà, le nombre de salles nécessaire). Cependant cette dernière dépend aussi de la variabilité intra-bande, la libération de la salle étant conditionnée par la durée de présence des animaux les moins performants de la bande. Ainsi, la rotation des bandes de porcs dans les salles conduit à faire partir les porcs les plus légers de chaque bande dans un laps de temps maximal déterminé par la configuration des bâtiments.

Une alternative à l'abattage des porcs légers en deçà du poids de carcasse minimal valorisé par la grille de paiement consiste à poursuivre leur élevage dans une salle annexe, mais en aucun cas (pour des raisons sanitaires) à les mélanger avec le groupe de porcs plus jeunes de la bande suivante. Les possibilités de tri sont aussi d'autant plus aisées que les bandes se succèdent à intervalles de temps rapprochés (conduites de pas hebdomadaire, en 20 ou 21 bandes).

Quoi qu'il en soit, dans la réalité des situations d'élevage, cette gestion des fins de bandes est souvent contrainte, les capacités et la compartimentation des bâtiments n'ayant pas toujours pu être adaptées aux évolutions de l'élevage (éventuelle modification de dimension ou de conduite, et surtout amélioration des performances...). On observe ainsi que l'ensemble des pratiques de rationnement ou de gestion des bandes ne permet pas de s'affranchir totalement de la variabilité des poids d'abattage. $\mathrm{Au}$ poids moyen de 91,4 kg relevé par Uniporc-Ouest en 2009 dans l'ensemble des élevages de sa zone d'action (plus de $80 \%$ des porcs français), cette variabilité conduit à une moins-value moyenne d'environ 2 centimes d' $€ / \mathrm{kg}$, et correspond à un ET intra-lot abattu d'environ $10 \mathrm{~kg}$ (figure $5 \mathrm{~b}$ ). On observe sur la même figure qu'une action de l'éleveur en faveur d'une réduction de la variabilité (réduction de l'ET de 10 à $5 \mathrm{~kg}$ ) améliore le résultat d'environ 2,5 centimes d' $€ / \mathrm{kg}$ (de façon variable toutefois selon le poids moyen).

c) Gestion des carcasses selon leur teneur en viande

De même que pour le poids de carcasse, le barème de paiement en vigueur incite fortement à la progression de la teneur en muscle, ce qui a été observé. Ce dispositif a ainsi motivé le recours massif des éleveurs à des types génétiques mâles très conformés (Piétrain) et incite à des pratiques de conduite amé- 
Figure 6. Plus-value moyenne par lot selon le Taux de Muscle des Pièces (TMP) moyen du lot, et la variabilité intra-lot (ET : Ecart-Type) de la TMP.

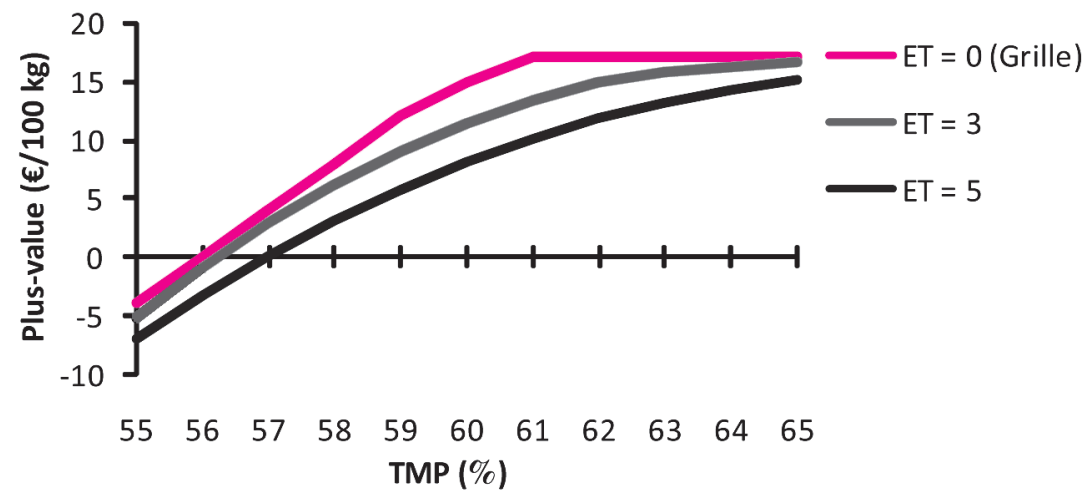

liorant ce paramètre. La variabilité intra-lot abattu est d'environ 3 points ; il en résulte que la courbe de la plus-value réelle par élevage reste très incitative à l'augmentation de la valeur moyenne du TMP jusqu'à des niveaux très élevés (figure 6).

Ce dispositif incite aussi à réduire la variabilité du TMP, celle-ci ayant en elle-même une incidence économique défavorable, illustrée sur la même figure par la comparaison entre deux niveaux de variabilité intra-lot (respectivement des ET de 3 et 5 points de TMP). Pour autant, sa maîtrise n'est pas totalement atteinte : on observe ainsi dans la zone Uniporc-Ouest en 2009 un écart moyen d'environ 2 points entre femelles et mâles castrés qui rend compte de la difficulté à séparer les animaux de sexes différents dans les conditions habituelles d'élevage et/ou à leur appliquer des modalités de rationnement différenciées, difficulté dont l'incidence est d'autant plus marquée que le TMP moyen est faible.

\section{3 / Variabilité des caracté- ristiques des truies}

La variabilité de l'état des truies à la mise bas résulte à la fois de la variabilité des réserves au début de la gestation et de celle du développement des compartiments corporels pendant la gestation. La première composante dépend bien souvent du déficit nutritionnel subi pendant la lactation précédente tandis que la seconde dépend directement de la quantité d'aliment allouée pendant la gestation et dans une moindre mesure des conditions de logement. Les travaux menés par l'INRA depuis une vingtaine d'années permettent désormais d'estimer les besoins de la truie gravide pour son entretien, le développement de la portée et de ses réserves. En outre, l'estimation factorielle des besoins (Dourmad et al 2005) permet également de prendre en compte le mode de logement de la truie et ses conséquences sur les besoins liés à l'activité physique ou à la thermorégulation. Il est également possible d'estimer de façon dynamique les besoins en fonction du stade de gestation.

\section{1 / Pendant la gestation}

a) Evolution de la variabilité de l'épaisseur de lard

A partir des mesures systématiques réalisées dans la station expérimentale de l'IFIP à Romillé, il apparaît que l'Epaisseur de Lard Dorsal (ELD) augmente de 16 à $20 \mathrm{~mm}$ en moyenne et la variabilité exprimée sur la base du $\mathrm{CV}$ est en moyenne de 15 et $14 \%$, respectivement à l'insémination et avant la mise bas pour le même jeu de données. Malgré la distribution d'une ration individualisée adaptée aux besoins, l'écart-type de l'ELD à l'entrée en maternité est donc en moyenne plus élevé de $0,3 \mathrm{~mm}$ qu'en début de gestation (figure 7). Cette augmentation sera d'autant plus importante qu'il y a une compétition à l'auge lors des repas. C'est notamment le cas quand les truies sont logées en petits groupes, non bloquées en réfectoires pendant les repas. Cela illustre l'évolution potentielle des difficultés de gestion des réserves Romillé entre 1998 et 2007). générées par le passage des truies d'un mode de logement individuel à un mode de logement en groupe sans gestion individualisée de l'alimentation. Une plus grande variabilité est également observée quand la proportion de jeunes truies dans les bandes est élevée et qu'elles sont toutes alimentées de la même façon, sans aucune correction selon leur gabarit ni leur ELD en début de gestation. Chez ces truies, la variabilité (écart-type) augmente en moyenne de $0,4 \mathrm{~mm}$ pendant la gestation.

b) Problèmes posés par les truies trop grasses à la mise bas

Les truies qui présentent des mises bas difficiles sont en moyenne plus lourdes et plus grasses que celles à mise bas sans problème. Elles sont également plus sujettes à des troubles locomoteurs et urinaires, qui résultent en partie de leur gabarit (Micquet et al 1990). Les truies les plus grasses à la mise bas présentent par ailleurs un appétit plus faible que les autres pendant la lactation (Dourmad 1989, EDE Bretagne 1995). Elles subissent alors, du fait de ce niveau d'ingestion moindre, un déficit nutritionnel plus important pendant la lactation qui est compensé par la mobilisation plus intense de leurs réserves corporelles. Or, la fonte des réserves corporelles lipidiques et protéiques, est l'un des facteurs majeurs impliqués dans les problèmes de reproduction après sevrage (Whittemore et Morgan 1990, Koketsu et al 1996, Quesnel et al 2008). Il faut noter que dans les études ci-dessus, les truies qualifiées de grasses présentaient une ELD supérieure à $25 \mathrm{~mm}$, ce qui est observé de plus en plus rarement dans les troupeaux actuellement.

c) Problèmes posés par les truies trop maigres à la mise bas

Les truies qui entrent trop maigres en maternité posent d'autres problèmes. Ceux-ci ne concernent pas le déroule-

Figure 7. Ecart-Type (ET) de l'épaisseur de lard dorsal mesurée sur des bandes de 24 truies 7 jours après l'insémination et son évolution jusqu'à l'entrée en maternité à 108 jours de gestation (données recueillies sur 118 bandes à la station IFIP de

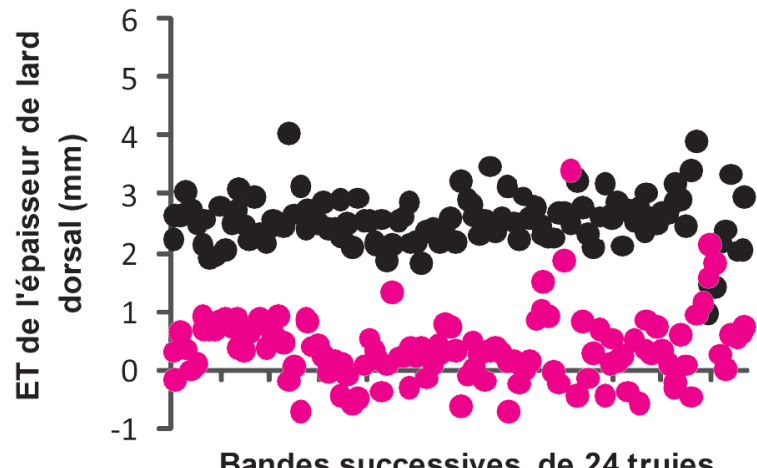

- 7 j après

l'insémination

évolution pendant la gestation 
Figure 8. Variabilité de la production laitière évaluée via la vitesse de croissance de

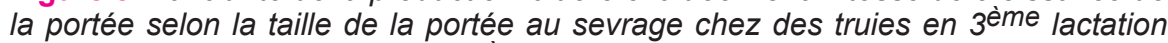
$(n=571,3,00 \pm 0,42 \mathrm{~kg} / \mathrm{j})$ et en $4^{e ̀ m e}$ lactation $(n=439,2,93 \pm 0,43 \mathrm{~kg} / \mathrm{j})$ étudiées à la station IFIP de Romillé.

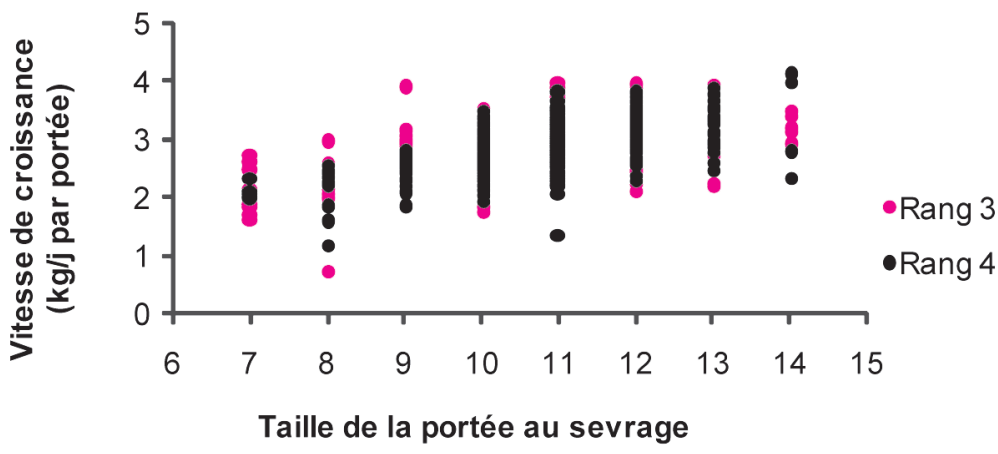

ment de la mise bas per se. Les observations réalisées en élevage mettent toutefois en évidence un poids de naissance moyen plus faible. Même si les truies les plus maigres ingèrent en moyenne plus d'aliment que les autres pendant la lactation, la quantité de nutriments disponibles (ingérés ou mobilisés à partir des réserves corporelles) n'est pas suffisante pour couvrir les besoins induits par la production de lait. Ces truies sont donc également en situation de déficit nutritionnel. Compte tenu du faible niveau de leurs réserves corporelles, une moindre production de lait est observée après la mise bas (O'Grady et al 1973). Au sevrage, les porcelets sont légers et la truie encore plus maigre.

d) Gestion de la variabilité pendant la gestation

Sur la base des connaissances disponibles et des outils associés (logiciel InraPorc $\AA$ ), une approche individualisée de la conduite alimentaire de la truie gestante a été développée ces dernières années. Le calcul d'apports individualisés se fonde sur un état initial de la truie défini par son âge, son poids et son ELD. Une quantité globale d'énergie est alors allouée à la truie sur les 115 jours de gestation afin de lui permettre d'atteindre des objectifs de poids (fonction de l'âge) et d'ELD à la mise bas. Puis, cet apport est modulé sur la durée de la gestation pour prendre en compte l'évolution exponentielle des besoins pour le développement de la portée (SalmonLegagneur 1965, Noblet et al 1985).

L'estimation du besoin énergétique repose donc sur des hypothèses d'augmentation du poids et de l'ELD de la truie pendant la gestation. Or, le gain de poids reflète principalement l'augmentation de la masse musculaire tandis que l'augmentation d'ELD reflète celle de la masse adipeuse. Cela revient donc à s'appuyer sur des hypothèses de composition du gain de poids. L'application de cette méthode devrait en théorie per- de portée allaitée donnée) et celle de la quantité de lait disponible par porcelet (qui dépend de la taille de la portée et de la variabilité de la production d'une tétine à l'autre) contribuent également à accroître la variabilité du poids des porcelets au sevrage dans l'élevage. La quantité de lait produite est estimée le plus souvent par une méthode indirecte, à partir de la vitesse de croissance de la portée et de sa taille (Noblet et Etienne 1989), les effets de la taille et du rang de portée sur la production laitière ayant déjà été décrits par ailleurs (Renaudeau 2001, Ngo et al 2012). Pour une taille et un rang de portée donnés, il apparaît que la production laitière estimée à partir de la croissance de la portée peut varier du simple au double (figure 8).

mettre d'homogénéiser l'état des truies à la mise bas. En réalité, il est sans doute plus exact de dire qu'elle permet de ne pas accroître voire de limiter l'augmentation de la variabilité de l'ELD au cours de la gestation. En effet, il est fréquent d'observer un gain de poids plus important que celui qui était prévu et une ELD moindre à la mise bas. La répartition de l'énergie déposée s'effectue donc souvent différemment de l'hypothèse retenue en moyenne pour le troupeau, ce qui contribue à maintenir une variabilité d'état entre les truies même quand les apports nutritionnels sont ajustés individuellement.

\section{2 / Pendant la lactation}

\section{a) Variabilité de la production laitière}

Dans la première partie de cet article, le poids de naissance est décrit comme l'un des facteurs explicatifs de la variabilité des performances du porc en croissance. La variabilité de la production laitière entre truies (pour une taille

\section{b) Variabilité de l'appétit}

Pour une production de lait donnée, la consommation spontanée d'aliment peut varier également du simple au double (figure 9). Une partie de ces écarts d'ingestion est attribuée au rang de portée des truies et à la taille de portée allaitée (Quiniou et al 1998). Une autre partie de cette variabilité semble également imputable à une sensibilité individuelle variable des truies à l'exposition au chaud. Or, dans les maternités, la température ambiante est la plupart du temps supérieure à la température de confort de la truie, ce qui peut donc accentuer l'écart d'ingestion entre les animaux.

\section{c) Gestion de la variabilité pendant la lactation}

La variabilité de la production laitière induit une variabilité des besoins nutritionnels de la truie. Pendant la lactation, un seul aliment est utilisé le plus souvent. Cet aliment doit permettre un

Figure 9. Variabilité des niveaux de production laitière (estimée à partir de la croissance de la portée) et d'ingestion spontanée d'aliment illustrée à partir de truies en 1 ère, $2^{\text {ème }}$ ou 4 ème lactation étudiées à la station IFIP de Romillé.

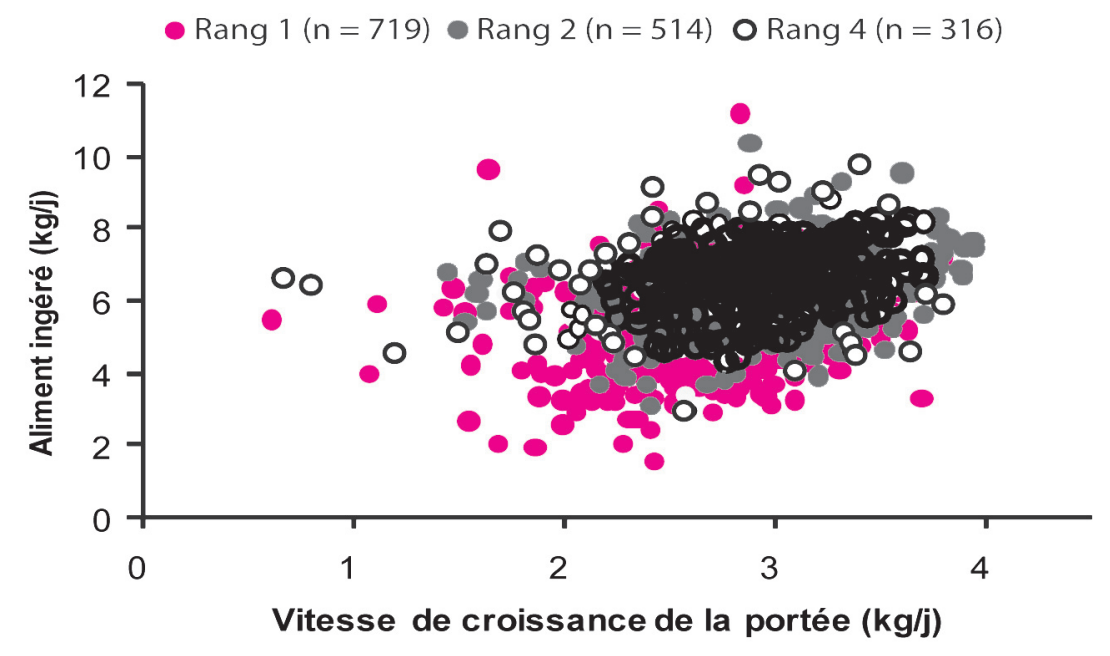


apport journalier, notamment en acides aminés, le plus proche possible des besoins alors que ces derniers varient considérablement d'une truie à l'autre. Par ailleurs, la variabilité du niveau d'ingestion implique que, pour une teneur donnée en nutriments dans l'aliment, l'apport quotidien en nutriments sera également très différent.

L'approche conseillée pour choisir le niveau de formulation en acides aminés consiste à calculer les besoins moyens de la population de truies de manière à préserver les réserves musculaires (Dourmad et al 2007). Dans cette approche, qui combine à la fois des considérations liées au système de distribution d'aliment en maternité et des considérations économiques liées au prix de l'aliment, le niveau de formulation n'est donc pas ajusté pour satisfaire les truies de la bande présentant le besoin le plus élevé. En pratique, il est toutefois difficile d'éviter complètement une mobilisation des réserves en muscle même lorsque les besoins en acides aminés sont couverts ; la mobilisation de ce compartiment contribue en effet en partie à compenser le déficit énergétique auquel la truie est soumise chroniquement pendant la lactation. Dans cette situation, la mobilisation du muscle conduit à une libération d'acides aminés qui constituent une réserve éventuellement utilisable par les truies pour lesquelles le niveau de formulation pourrait être insuffisant.

\section{Conclusion}

Dans les élevages de production, les porcs sont issus de différents croisements génétiques et différents types sexuels qui peuvent expliquer des écarts de performances moyennes (Agence de la Sélection Porcine 2007). La variabilité du poids à la naissance entraîne des taux de pertes importants, observés principalement pour les porcelets les plus chétifs de la portée ; elle est désormais prise en compte en sélection. Dans l'attente d'une amélioration de ce critère en élevage, la variabilité de poids, existant entre les porcs complique la conduite, tant au niveau de la gestion des transitions alimentaires que du tri des porcs avant le départ à l'abattoir. Du côté des truies, la variabilité du potentiel laitier et de la prise alimentaire pendant la lactation complique également la conduite de l'alimentation tant pendant la gestation que pendant la lactation. Au cours de ces dernières années, l'éleveur a été contraint de s'adapter et de mettre en place de nouvelles stratégies afin de rentabiliser au mieux économiquement ses animaux, très différents les uns des autres au sein d'une même population.

L'élaboration des plans d'alimentation, tant pour les animaux en croissance que pour les reproducteurs, se fonde désormais sur une approche analytique très poussée ; cependant, si un modèle tel InraPorc ${ }^{\circledR}$ la formalise et ouvre la voie à une gestion individualisée des animaux, sa mise en œuvre en élevage reste parfois difficile pour des raisons pratiques. La mise au point d'équipements et d'automatismes à l'interface des résultats de la recherche et de leur application en élevage de production est un des éléments qui rendra opérationnel le concept d'élevage de précision.

La variabilité au sein de cohortes d'animaux doit être prise en compte, à la fois pour déterminer des stratégies réellement optimisées à l'échelle de ces cohortes et, au-delà, pour maîtriser ou réduire la variabilité elle-même. La gestion de cette variabilité est clairement perçue par de nombreux éleveurs comme un élément de progrès de l'efficacité technique. C'est vrai en ce qui concerne les caractéristiques des truies en général et les poids des porcs à un âge donné. A contrario, la situation est sans doute moins claire en ce qui concerne la composition corporelle des porcs à l'abattage. Dans le contexte actuel où les modes de production n'ont pas été davantage spécialisés, la variabilité de la teneur en muscle des carcasses, qui est reprochée aux éleveurs et qui les pénalise économiquement, est pourtant l'un des éléments permettant aux opérateurs industriels en aval de l'élevage de trouver les matières premières adaptées à la diversité des produits (frais, produits cuits, produits secs) demandés par le marché.

\section{Références}

Agence de la Sélection Porcine, 2007. Résultats du $28^{\text {ème }}$ test de contrôle des produits terminaux issus de différentes combinaisons de types génétiques de truies et de verrats parentaux agréés par le Ministère chargé de 1'Agriculture. TechniPorc, 30, 7-20.

Anil L., Anil S., Deen J., 2007. Effects of allometric space allowance and weight group composition on grower-finisher pigs. Can. J. Anim. Sci., 87, 139-151.

Beaulieu A.D., Aalhus J.L., Williams N.H Patience J.F., 2010. Impact of piglet birth weight, birth order, and litter size on subsequent growth performance, carcass quality, muscle composition, and eating quality of pork. J. Anim. Sci., 88, 2767-2778.

Boulot S., Dubroca S., Badouard B., 2005. Gestion pharmacologique de la reproduction le point sur les pratiques des éleveurs. TechniPorc, 28, 9-12.

Brossard L., Dourmad J.Y, Rivest J., van Milgen J., 2009. Modelling the variation in performance of a population of growing pig as affected by lysine supply and feeding strategy. Animal, 3, 1114-1123.

Brossard L., Meunier-Salaün M.C., Dourmad J.Y., Lescoat P., Gardeur J.N., 2011. Évolution de la variabilité du poids chez les groupes de porcs en croissance selon la variabilité initiale et la densité d'élevage. Journ. Rech. Porcine, 43, 179-180.

Brossard L., Quiniou N., Dourmad J.Y., van Milgen J., 2012. Prise en compte de la variabilité individuelle dans l'estimation des besoins nutritionnels et la modélisation de la réponse des porcs en croissance aux apports alimentaires. INRA Prod. Anim., 25, 17-28.

Brouns F., Edwards S.A., 1994. Social rank and feeding behavior of group housed sows fed competitively or ad libitum. Appl. Anim. Behav. Sci., 39, 225-235.

Bruininx E.M., van der Peet-Schwering C.M., Schrama J.W., Vereijken P.F., Vesseur P.C., Everts H., den Hartog L.A., Beynen A.C 2001. Individually measured feed intake characteristics and growth performance of group-housed weanling pigs: effects of sex initial body weight, and body weight distribution within groups. J. Anim. Sci., 79, 301308 .

Campos P.H.R.F., Silva B.A.N., Donzele J.L., Oliveira R.F.M., Knol E.F., 2011 Effects of sow nutrition during gestation on within-litter birth weight variation: a review. Animal, doi: 10.1017/S17517311 11002242
Corpen 2003. Estimation des rejets d'azote, phosphore, potassium, cuivre et zinc des porcs, Corpen Edition, Paris, France, 41p.

Damgaard L.H., Rydhmer L., Løvendahl P., Grandison K., 2003. Genetic parameters for within-litter variation in piglet birth weight and change in within-litter variation during suckling. J. Anim. Sci., 81, 604-610.

Dourmad J.Y., 1989. Influence du niveau alimentaire de gestation sur les performances de reproduction et le comportement alimentaire de la truie en lactation. Journ. Rech. Porcine Fr., 21, 109-114

Dourmad J.Y., Etienne M., Noblet J., Valancogne A., Dubois S., van Milgen J., 2005. InraPorc: un outil d'aide à la décision pour l'alimentation des truies reproductrices. Journ. Rech. Porcine, 37, 299-306.

Dourmad J.Y., Etienne M., Valancogne A., Dubois S., van Milgen J., Noblet J., 2007. InraPorc: A model and decision support tool for the nutrition of sows. Anim. Feed Sci. Tech., $143,372-386$.

EDE Bretagne, 1995. Conduite alimentaire des truies en maternité. Rapport d'étude, Chambre d'Agriculture Régionale de Bretagne, Rennes, France, 45p. 
Edwards S.A., Malkin S.J., 1986. An analysis of piglet mortality with behavioural observations. Anim. Prod., 42, 470.

English P.R., Wilkinson V., 1982. Management of the sow and litter in late pregnancy and lactation in relation to piglet survival and growth. In: Control of pig reproduction. Cole D.J.A., Foxcroft G.R. (Eds), Butterworths, London, UK, 479-506.

Fix J.S., Cassady J.P., Holl J.W., Herring W.O., Culbertson M.S., See M.T., 2010. Effect of piglet birth weight on survival and quality of commercial market swine. Livest. Sci., 132, 98-106.

Garreau H., Bolet G., Larzul C., RobertGranié C., Saleil G., SanCristobal M., Bodin L., 2008. Results of four generations of a canalising selection for rabbit birth weight. Livest. Sci., 119, 55-62.

Gestion Technique des Troupeaux de TruiesIFIP, 2011. Le porc par les chiffres. Les résultats nationaux, régionaux et départementaux de Gestion Technique des Troupeaux de Truies, Gestion-Technico-Économique des élevages de porcs. IFIP Edition, Paris, France, 44p.

Gondret F., Lefaucheur L., Louveau I., Lebret B., Pichodo X., Le Cozler Y., 2005. Influence of piglet birth weight on postnatal growth performance, tissue lipogenic capacity and muscle histological traits at market weight. Livest. Prod. Sci., 93, 137-146.

Gondret F., Lefaucheur L., Juin H., Louveau I., Lebret B., 2006. Low birth weight is associated with enlarged muscle fibre area and impaired meat tenderness of the longissimus muscle in pigs. J. Anim. Sci., 84, 93-103.

Gondret F., Perruchot M.H., Tacher S. Bérard J., Bee G., 2011. Differential gene expressions in subcutaneous adipose tissue pointed to a delayed adipocytic differentiation in small pig fetuses compared to their heavier siblings. Differentiation, 81, 253-260

Gourmelen C., Le Moan L., 2004. Impact économique de l'hyperprolificité en élevage de production - Comparaison de 11 scenarii. Journ. Rech. Porcine, 36, 463-470.

Hauschild L., Lovatto P.A., Pomar J., Pomar C., 2010. Estimation journalière des besoins nutritionnels individuels des porcs en croissance en fonction de leur parcours de croissance et de consommation. Journ. Rech. Porcine, 42, 99-105.

Herpin P., Le Dividich J., Hulin J.C., Fillaut M., de Marco F., Bertin R., 1996. Effects of the level of asphyxia during delivery on viability at birth and early postnatal vitality of newborn pigs. J. Anim. Sci., 74, 2067-2075.

Huby M., Canario L., Tribout T., Caritez J.C., Billon Y., Gogué J., Bidanel J.P., 2003. Genetic correlations between litter size and weights, piglet weight variability and piglet survival from birth to weaning in Large White pigs. EAAP Meeting, Roma, Italy. 362.

IFIP, 2008. L'alimentation de la truie. IFIP Edition, Paris, France, 44p.

Kammersgaard T.S., Pedersen L.J. Jørgensen E., 2011. Hypothermia in neonatal piglets: interactions and causes of individual differences. J. Anim. Sci., 89, 2073-2085.

Knol E.F., Leenhouwers J.I., van der Lende T., 2002. Genetic aspects of piglet survival. Livest. Prod. Sci., 78, 47-55.

Koketsu Y., Dial G.D., Pettigrew J.E., King V.L., 1996. Feed intake pattern during lactation and subsequent reproductive performance of sows. J. Anim. Sci., 74, 2875-2884.

Le Cozler Y., Pellois H., Dagorn J., Guyomarc'h C., Quinio P.Y., Pichodo X., 2000. Importance et origine des porcelets mort-nés. Chambre d'Agriculture Régionale de Bretagne, Rennes, France, 77p.

Le Cozler Y., Pichodo X., Roy H., Guyomarc'h C., Quiniou N., Louveau I., Lebret B., Lefaucheur L., Gondret F., 2004. Influence du poids et de la taille de la portée à la naissance sur la survie du porcelet, ses performances de croissance, d'abattage et la qualité de la viande. Journ. Rech. Porcine, 36, 443-450.

Leenhouwers J.I., van der Lende T., Knol E.F., 1999. Analysis of stillbirth in different lines of pig. Livest. Prod. Sci., 57, 243-253.

Lösel D., Kalbe C., Rehfeldt C., 2009 L-Carnitine supplementation during suckling intensifies the early postnatal skeletal myofibe formation in piglets of low birth weight. J. Anim. Sci., 87, 2216-2226.

Lynch P.B., Cahill A, Lawlor P., Boyle L., O'Doherty J.V., Le Dividich J., 2006. Studies on growth rates in pigs and the effect of birth weight. Report RMIS No. 5220 Teagasc, Agric. Food Dev. Authority (Eds), Moorepark, Fermoy, Co. Cork, 51pp http://www.teagasc.ie/research/reports/pigs/52

\section{0/eopr-5220.htm}

Manuel d'application du Guide de bonnes pratiques d'hygiène en élevage de porcs, 2009 Chapitre 2c. Conduite d'élevage : soins aux truies et aux porcelets en maternité. IFIP Edition, Paris, France, 27-28.

Marchant J.N., Rudd A.R., Mendl M.T. Broom D.M., Meredith M.J., Corning S. Simmins P.H., 2000. Timing and causes of piglet mortality in alternative and conventional farrowing systems. Vet. Rec., 147, 209-214.

Marouby H., Daridan D., van Ferneij J.P., Badouard B., Salaün Y., 2001. Le prix du porc perçu par les producteurs dans les principaux bassins de l'Union Européenne. Journ. Rech. Porcine Fr., 33, 241-248.

Martel G., Dourmad J.Y., Dedieu B., 2008 Do labour productivity and preferences about work load distribution affect reproduction management and performance in pig farms? Livest. Sci., 116, 96-107.

Massiot A., Quiniou N., 2008. Conséquence du sexage ou non des porcs et de la période de rationnement sur les performances en engraissement. Journ. Rech. Porcine, 40, 209-212.

Micquet J.M., Madec F., Paboeuf F., 1990. Epidémiologie des troubles de la mise bas chez la truie : premiers résultats d'une étude réalisée dans deux élevages. Journ. Rech. Porcine Fr. $22,325-332$.

Milligan B.N., Fraser D., Kramer D.L. 2002. Within-litter birth weight variation in the domestic pig and its relation to pre-weaning survival, weight gain, and variation in weaning weights. Livest. Prod. Sci., 76, 181-191.

Morise A., Sève B., Macé K., Magliola C. Le Huërou-Luron I., Louveau I., 2009. Impact of intrauterine growth retardation and early protein intake on growth, adipose tissue, and the insulin-like growth factor system in piglets. Pediatr. Res., 65, 45-50.

Ngo T.T., Quiniou N., Heugebaert S., Paboeuf F., Dourmad J.Y., 2012. Influence du rang de portée et du nombre de porcelets allai- tés sur la production laitière des truies, Journ. Rech. Porcine, 44, 195-196.

Noblet J., Etienne M., 1989. Estimation of sow milk nutrient output. J. Anim. Sci., 67, 3352-3359.

Noblet J., Quiniou N., 1999. Principaux facteurs de variation du besoin en acides aminés du porc en croissance. TechniPorc, 22, 9-16.

Noblet J., Close W.H., Heavens R.P., 1985. Studies on the energy metabolism of the pregnant sow. 1. Uterus and mammary tissue development. Brit. J. Nutr., 53, 251-265.

O'Grady J.F., Elsley F.W.H., MacPherson R.M., McDonald I., 1973. The response of lactating sows and their litter to different dietary energy allowances. 1. Milk yield and composition, reproductive performance of sows and growth rate of litters. Anim. Prod., 17, 65-74.

Orgeur P., Le Dividich J., Colson V., Meunier-Salaün M.C., 2002. La relation mèrejeune chez les porcins : de la naissance au sevrage. INRA Prod. Anim., 15, 185-198.

Petit Robert, 1990. Dictionnaire de la langue française. Ed. Dictionnaires le Robert, Paris, France, 1218 et 2641.

Quesnel H., Brossard L., Valancogne A., Quiniou N., 2008. Influence of some sow characteristics on within-litter variation of piglet birth weight. Animal, 2, 1842-1849.

Quiniou N., 2005. Influence de la quantité d'aliment allouée à la truie en fin de gestation sur le déroulement de la mise bas, la vitalité des porcelets et les performances de lactation. Journ. Rech. Porcine, 37, 187-194.

Quiniou N., Dourmad J.Y., Noblet J., 1998. Facteurs de variation de l'appétit des truies en lactation. INRA Prod. Anim., 11, 247-250.

Quiniou N., Dagorn J., Gaudré D., 2002. Variation of piglets' birth weight and consequences on subsequent performance. Livest. Prod. Sci., 78, 63-70.

Quiniou N., Gaudré D., Pichodo X., Le Cozler Y., 2004. Caractérisation de l'indice de consommation pendant l'engraissement selon le poids des porcelets à la naissance Journ. Rech. Porcine, 36, 403-408.

Quiniou N., Richard S., Mourot J., Etienne M., 2008. Effect of dietary fat or starch supply during gestation and/or lactation on the performance of sows, piglets' survival and on the performance of progeny after weaning. Animal, 2, 1633-1644.

Rehfeldt C., Tuchscherer A., Hartung M., Kuhn G., 2008. A second look at the influence of birth weight on carcass and meat quality in pigs. Meat Sci., 78, 170-175.

Renaudeau D., 2001. Adaptation nutritionnelle et physiologique aux températures ambiantes élevées chez la truie en lactation. Thèse, Université Rennes I, Rennes, France, 160p.

Rivest J., 2009. Etude des impacts zootechniques et économiques d'une augmentation du poids d'abattage chez le porc. Thèse, Université Laval, Québec, 241p.

Salmon-Legagneur E., 1965. Quelques aspects des relations nutritionnelles entre la gestation et la lactation chez la truie. Ann. Zootech., 14, 1-137.

Sasaki Y., Koketsu Y., 2007. Variability and repeatability in gestation length related to litter performance in female pigs on commercial farms. Theriogen., 68, 123-127. 
Scheel D.E., Graves H.B., Sherritt G.W., 1977. Nursing order, social dominance and growth in swine. J. Anim. Sci., 45, 219 229.

van den Brand H., Soede N.M., Kemp B., 2006. Supplementation of dextrose to the diet during the weaning to estrus interval affects subsequent variation in within-litter piglet birth weight. Anim. Reprod. Sci., 91, 353-358.

Whittemore C.T., Morgan C.A., 1990. Model components for the determination of energy and protein requirements for the breeding sows: a review. Livest. Prod. Sci., 26, 1-37.

Wittenburg D., Guiard V., Teuscher F., Reinsch N., 2011. Analysis of birth weight variability in pigs with respect to liveborn and total born offspring. J. Anim. Breeding Genet., $128,35-43$.

Zaleski H.M., Hacker R.R., 1993. Effect of oxygen and neostigmine on stillbirth and pig viability. J. Anim. Sci., 71, 298-305.

\section{Résumé}

La variabilité de la prolificité est maîtrisable par l'éleveur tant que le nombre de porcelets nés vivants n'excède pas le nombre de tétines fonctionnelles dans la maternité : des adoptions entre portées permettent alors d'ajuster le nombre de porcelets allaités aux capacités laitières des truies. Quand la prolificité est très élevée, la gestion des porcelets devient plus compliquée et la variabilité de leurs poids de naissance pénalise les chances de survie des individus les plus petits. S'ensuit aussi une variabilité accrue des performances de croissance. Des solutions à court terme peuvent être mises en ouvre, en particulier l'adaptation selon le poids des animaux des soins pratiqués aux porcelets à la naissance, la constitution de lots de porcs sur la base du poids au sevrage et à l'entrée en engraissement, et l'adaptation des transitions alimentaires en fonction du poids moyen de chaque lot. Malgré ces pratiques, la variabilité des poids au sein d'une bande persiste jusqu'en fin d'engraissement, compliquant l'organisation des départs pour l'abattoir. Ceux-ci sont généralement gérés au mieux de l'intérêt économique de l'éleveur en relation avec la grille de paiement basée en partie sur le poids de carcasse. Sans attendre les progrès de la sélection génétique pour limiter la variabilité des performances des truies et des porcelets, la prise en compte précoce des besoins individuels des truies au sein d'une bande permettrait de gérer l'évolution de l'adiposité maternelle pendant la gestation et faciliter le déroulement des mises bas. La prise en compte de l'évolution des besoins nutritionnels au cours de la gestation par des plans d'alimentation adaptés améliore la vitalité des nouveau-nés, mais jusque-là ne joue pas sur la variabilité des poids intra-portée. Ces améliorations sont obtenues le plus souvent dans des troupeaux expérimentaux, et restent à généraliser dans les élevages de production.

\section{Abstract}

Variability in pig units: description and on-farm implication

Pig breeders can cope with variation in sow prolificacy as long as the total number of piglets born alive does not exceed the total number of functional teats of the sows in the farrowing unit. Cross-fostering allows adjusting the size of the litter to the milk production potential of the sow. When prolificacy increases, this objective becomes more difficult to achieve. In addition, the within-litter variation of birth weight increases with prolificacy, compromising the survival chance of small piglets. Short-term solutions can be obtained by providing additional care around birth, by grouping of pigs of similar body weight and by providing appropriate feeding strategies. Nevertheless, variability is still observed at the end of the fattening period. This results in difficulties to find the best slaughtering strategy, especially when the economic return depends on a payment grid that is based partly on carcass weight. Although genetic selection may eventually reduce the variation in performance in sows and piglets, feeding strategies that account for the individual requirement of the gestating sow can already be used to improve the farrowing process. Although this helps to improve piglet vitality at birth, to date, it is not effective in reducing within-litter variation in birth weight. Most of these improvements have been reported for sows and piglets kept in experimental facilities. It remains a challenge to obtain these results in production units.

QUINIOU N., BROSSARD L., VAN MILGEN J., SALAÜN Y., QUESNEL H., GONDRET F., DOURMAD J.-Y., 2012. La variabilité des performances animales en élevage porcin : description et implications pratiques. INRA Prod. Anim. 25, 5-16. 